\section{Spatial distribution of hospital admissions diabetes mellitus in Espírito Santo state, Brazil}

\title{
Distribuição espacial das internações hospitalares por diabetes mellitus no Espírito Santo, Brasil
}

ABSTRACT | Introduction: Diabetes melittus is a metabolic disease featured by byperglycemia; it is associated with kidney and heart complications that increase the likelihood of diabetesassociated hospitalization by two to six times. Objective: Analyzing the spatial distribution of hospital admissions due to diabetes mellitus in Espirito Santo State, from 2011 to 2015. Method: Ecological study about diabetes-associated hospitalization of adult individuals living in different counties in Espirito Santo State was carried out from 2011 to 2015. Hospitalizations were featured based on absolute and relative frequencies. Subsequently, hospitalization rates were calculated and smoothed by global and local Bayesian estimators. Local Moran's Index was used to check local spatial correlation. Results: In total, 11,514 hospitalizations due to diabetes mellitus were reported in Espirito Santo State, from 2011 to 2015; The highest prevalence of hospitalizations was observed among 60-yearold (or older), brown and female individuals, - with a significant number of patients required emergency care. Iuna and São Roque do Canaa were the counties recording the bighest and lowest gross rates, respectively. After the Moran Global smoothing and local methods were applied, it was possible seeing that Irupi County and Vitória City presented the highest and the lowest diabetesassociated hospitalization rates, respectively. Conclusion: Based on the spatial analysis, it was possible identifying the counties in need tof expanding, structuring or improving their primary health - care services to enable specific planning focusing on reducing the number of hospitalizations due to diabetes.

Keywords | Diabetes Mellitus; Hospitalization; Descriptive Epidemiology; Spatial Analysis.
RESUMO| Introdução: O diabetes melittus (DM) é uma patologia metabólica caracterizada por hiperglicemia associada às complicações de alguns órgãos, como rins e coração, as quais aumentam de duas a seis vezes a probabilidade de internação por diabetes. Objetivo: Analisar a distribuição espacial das internações hospitalares por DM no estado do Espirito Santo (ES), Brasil, no período de 2011 a 2015. Método: Estudo ecológico das internações hospitalares por diabetes em adultos residentes nos municípios do ES. As internações foram caracterizadas por meio de frequências absolutas e relativas. Sequencialmente, as taxas de internações foram calculadas e suavizadas pelos estimadores bayesianos globais e locais. Para verificar a correlação espacial local, aplicou-se o Índice de Moran Local. Resultados: No período de 2011 a 2015 foram notificadas 11.514 internações por DM no ES, tendo maior prevalência no sexo feminino, na raça/cor parda e na faixa etária $\geq 60$ anos, apresentando ainda um número expressivo no caráter de atendimento de urgência. Os municípios com maior e menor taxa bruta foram Iúna e São Roque do Canaã, respectivamente. Após a utilização dos métodos de suavização de Moran Global e local, identificou-se que Irupi apresentou a maior taxa de internação por diabetes, enquanto Vitória representou o município com menor taxa de internação hospitalar por diabetes. Conclusão: Com a análise espacial é possível verificar os municípios que necessitam de ampliação, estruturação ou aperfeiçoamento dos serviços de atenção primária à saúde, o que permite um planejamento especifico para redução de internações por diabetes.

Palavras-chave| Diabetes Mellitus; Hospitalização; Epidemiologia Descritiva; Análise Espacial.

${ }^{1}$ Escola Nacional de Saúde Pública Sérgio Arouca, Fundação Oswaldo Cruz. Rio de Janeiro/RJ, Brasil. ${ }^{2}$ Universidade Federal do Espírito Santo. Vitória/ES, Brasil.

${ }^{3}$ Universidade de São Paulo. São Paulo/SP, Brasil. 


\section{INTRODUÇÃO|}

Atualmente, o diabetes mellitus (DM) representa uma importante causa de morbimortalidade, sendo responsável por quase quatro milhões de mortes entre indivíduos de 20 a 79 anos, o equivalente a $10,7 \%$ da mortalidade mundial por todas as causas nessa faixa. Estima-se ainda que 425 milhões de pessoas vivam com DM, ou seja, 8,8\% da população mundial com projeções de 629 milhões de casos até $2045^{1}$.

Esse agravo se dá por defeitos de secreção e/ou ação da insulina envolvendo processos patogênicos específicos, como a destruição das células produtoras de insulina, distúrbios da secreção da insulina, resistência à ação da insulina, entre outros ${ }^{2}$.

O Brasil ocupa a $4^{\text {a }}$ colocação entre os 10 países com maior carga de DM, com aumento dos casos em 61,8\% no período de 2006 a 2016. Observou-se ainda que Rio de Janeiro (10,4\%), Belo Horizonte (10,1\%), São Paulo (10\%) e Vitória $(9,7 \%)$ são as capitais com as maiores prevalências de $\mathrm{DM}$ do país ${ }^{3}$.

Além disso, o DM é a segunda maior causa de anos de vida produtivos perdidos devido à incapacidade (DALY), representando quase $6,1 \%$ da carga de doenças, com uma taxa de DALY de 9,2/100 mil habitantes ${ }^{4}$.

Quanto aos fatores que contribuem com o aumento da incidência e prevalência do DM destacam-se: sedentarismo, adoção de estilo de vida pouco saudável, dieta inadequada, obesidade, envelhecimento da população e urbanização crescente, atividade física insuficiente, tabagismo e uso nocivo de álcool ${ }^{5}$.

O DM é uma doença de alto custo social, financeiro e emocional para o indivíduo, além de onerar o Sistema Único de Saúde (SUS) devido às complicações e sequelas, como problemas cardiocirculatórios, insuficiência renal, cegueira, retinopatia diabética e até as amputações de extremidades ${ }^{6}$.

Apesar da gama de tratamentos eficazes disponibilizados para prevenir ou retardar as complicações agudas e crônicas que o DM gera no indivíduo, ainda existem pacientes que sofrem com o fardo gerado pela doença, suscitando num aumento significativo pela procura de cuidados de saúde ${ }^{4}$. Os altos índices de hospitalizações decorrentes de complicações e quadros clínicos descompensados de
DM geram uma demanda maior aos serviços de saúde e consequentemente aos custos sociais ${ }^{7}$.

O estudo realizado por Berardis em diferentes regiões da Itália verificou que 23,9\% dos indivíduos com DM já internaram em instituição hospitalar pelo menos uma vez, correspondendo a mais de 12.000 internações por 100 mil indivíduos/ano em média. Além disso, os autores identificaram que as complicações da doença aumentam de duas a seis vezes a probabilidade de hospitalização ${ }^{8}$.

Ademais, estudo realizado no Espírito Santo, no período de 2005 a 2009, apontou que o DM está entre as 10 causas mais frequentes de internações do Estado. Em quase todas as microrregiões do Estado, verificou-se aumento da proporção de internações relacionadas ao pré-natal e parto, DM e angina. Esse achado desperta preocupações por se tratar de internações por Condições Sensíveis à Atenção Primária (CSAP), cujo diagnóstico precoce e tratamento ambulatorial são efetivos para evitar hospitalização?. Destaca-se ainda que o DM é um agravo alvo de programas da Atenção Básica há muitos anos, e isso aumenta as preocupações sobre a assistência e o cuidado ofertado ${ }^{9,10 .}$

Diante desse contexto, postula-se que no ES a distribuição geográfica das internações por DM não ocorra de forma uniforme devido a diversos fatores organizacionais dos serviços de saúde. Dessa forma, haveria territórios em maior risco desse agravo, o que exige investigação mais detalhada, uma vez que o ES é caracterizado por diferentes níveis de desenvolvimento e organização dos serviços de saúde, especialmente da Atenção Primária à Saúde no que tange à Estratégia de Saúde da Família.

Isso posto, o objetivo deste estudo foi analisar a distribuição espacial das internações hospitalares por DM no estado do ES no período de 2011 a 2015.

\section{MÉTODOS |}

Estudo ecológico das internações hospitalares por DM em adultos residentes do Espírito Santo, no período de 2011 a 2015. O Espírito Santo possui 78 municípios, divididos em quatro regiões de saúde (Norte, Sul, Metropolitana e Central), com uma extensão territorial de 46.086,907 km² e população estimada de 4.016 .356 pessoas $^{11}$. 
Os dados foram obtidos do Sistema de Informação Hospitalar disponibilizado no DATASUS, sendo a população extraída do mesmo portal. Inicialmente foram estimadas as frequências absolutas e relativas das internações hospitalares de acordo com as seguintes variáveis de interesse: região de saúde; sexo; raça/cor; faixa etária; regime de internação; e caráter de atendimento. Para a análise, foi utilizado o software Microsoft Excel, versão 2010.

Em seguida, aplicou-se a análise espacial das internações hospitalares por DM, utilizando a malha digital do estado do Espírito Santo disponibilizada pelo Instituto Brasileiro de Geografia e Estatística (IBGE). Para essa análise, calcularam-se as taxas de internações hospitalares por DM de cada município do Espírito Santo no período de estudo, dividindo-se o total das internações hospitalares por município pela população residente de cada município e no mesmo período, multiplicado por 100 mil. Os dados foram computados e apresentados por meio de mapas, utilizando os softwares TerraView versão 4.2.2 e GeoDa versão 1.6.

Para corrigir as taxas epidemiológicas, empregou-se a análise espacial. O primeiro método aplicado foi o estimador bayesiano empírico global que tem como objetivo uma aproximação da taxa média do conjunto dos municípios. O segundo método foi o estimador bayesiano empírico local que trabalha com a média do coeficiente de internação encontrado por DM nos municípios vizinhos, por meio da expressão $\theta_{i}=p_{i} t_{i}+\left(1-p_{i}\right.$ ) $\mu_{i}$, onde $t_{i}$ representa o coeficiente de internação hospitalar por DM no Espírito Santo, $\mu_{\mathrm{i}}$ é a taxa global para o Estado, e pi consiste no peso entre 0 e 1 que depende do tamanho da população na área (i $)^{12}$.

Por fim, calculou-se o Índice Local de Moran I para analisar o nível de dependência espacial, ou seja, verificar se a distribuição das internações hospitalares por DM no espaço ocorre de forma aleatória ou segue algum padrão de ocorrência. Para isso, considerou-se o nível de significância de $5 \%{ }^{13}$.

Esta pesquisa utilizou dados de domínio público, portanto não houve a necessidade de submissão ao Comitê de Ética em Pesquisa.

\section{RESULTADOS |}

No período de 2011 a 2015 foram notificadas 11.514 internações por DM no Espírito Santo, sendo que a maioria ocorreu na região de saúde Sul (33,23\%). Quanto às características sociodemográficas, verificou-se maior frequência de internações hospitalares por DM no sexo feminino $(56,35 \%)$, raça/cor parda $(42,97 \%)$, e na faixa etária $\geq 60$ anos $(54,30 \%)$ (Tabela 1$)$.

Também observou-se que 56,72\% (6531) do total de internados por DM ocorreu em unidades de regime privado. Evidenciou-se ainda o número expressivo no quesito caráter de atendimento, onde 98,64\% (11.357) dos pacientes foram internados em caráter de urgência (Tabela 1).

Tabela 1 - Caracterização das internações hospitalares por diabetes mellitus. Espirito Santo, 2011-2015

\begin{tabular}{|c|c|c|c|}
\hline Variáveis & Categorias & $\mathbf{N}$ & $\%$ \\
\hline \multirow{4}{*}{ Região de Saúde } & Central & 2039 & 17,71 \\
\hline & Metropolitana & 3755 & 32,61 \\
\hline & Norte & 1894 & 16,45 \\
\hline & Sul & 3826 & 33,23 \\
\hline \multirow{2}{*}{ Sexo } & Masculino & 5026 & 43,65 \\
\hline & Feminino & 6488 & 56,35 \\
\hline \multirow{6}{*}{ Raça/Cor } & Branca & 3196 & 27,76 \\
\hline & Preta & 886 & 7,69 \\
\hline & Parda & 4947 & 42,97 \\
\hline & Amarela & 21 & 0,18 \\
\hline & Indígena & 2 & 0,02 \\
\hline & Sem informação & 2462 & 21,38 \\
\hline \multirow{5}{*}{ Faixa Etária } & 20 a 29 anos & 403 & 3,50 \\
\hline & 30 a 39 anos & 690 & 5,99 \\
\hline & 40 a 49 anos & 1415 & 12,29 \\
\hline & 50 a 59 anos & 2754 & 23,92 \\
\hline & $\geq 60$ anos & 6252 & 54,30 \\
\hline \multirow{3}{*}{$\begin{array}{l}\text { Regime de } \\
\text { Internação }\end{array}$} & Público & 4586 & 39,83 \\
\hline & Privado & 6531 & 56,72 \\
\hline & Ignorado & 397 & 3,45 \\
\hline \multirow{2}{*}{$\begin{array}{l}\text { Caráter do } \\
\text { Atendimento }\end{array}$} & Eletivo & 157 & 1,36 \\
\hline & Urgência & 11.357 & 98,64 \\
\hline Total & & 11.514 & 100,00 \\
\hline
\end{tabular}

Dentre os 78 municípios que compõem o Espírito Santo, o que apresentou maior taxa de internação bruta foi Iúna (388,30 por 100 mil habitantes), seguido de São José do Calçado (364,03 por 100 mil habitantes) e Bom Jesus do Norte (243,71 por 100 mil habitantes). Por outro lado, os municípios que apresentaram as menores taxas de internação foram São Roque do Canaã (11,50 por 100 mil habitantes), Governador Lindenberg (15,06 por $100 \mathrm{mil}$ habitantes) e Fundão (15,65 por 100 mil habitantes) (Figura 1 - mapa “a”). 
Figura 1 - Distribuição espacial das taxas de internações hospitalares por diabetes mellitus. Espirito Santo, 2011-2015
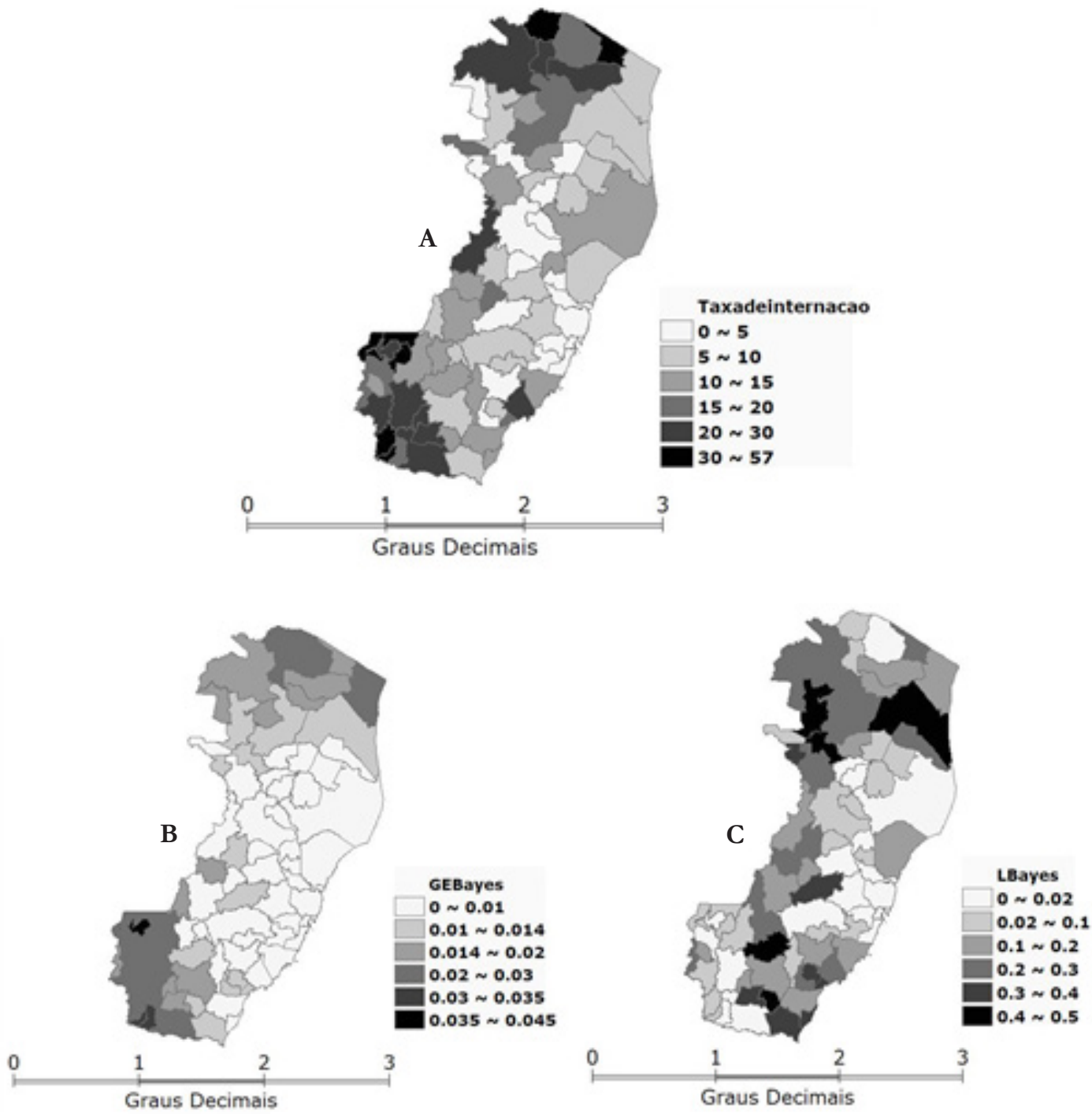

A: Taxa de Incidência Bruta. B: Estimador bayesiano empírico global. C: Estimador bayesiano empírico local.

Após a utilização do método de suavização de Moran Global, identificou-se que Irupi apresentou a maior taxa de internação por DM (0,04 por 100 mil habitantes), enquanto Vitória representou o município com a menor taxa de internação hospitalar por DM (0,004 por 100 mil habitantes) (Figura 1 - mapa "b”). Da mesma forma, após a aplicação do Índice de Moran Local, observou-se que Irupi e Vitória apresentaram a maior $(45,46$ por 100 mil habitantes) e menor (4,17 por 100 mil habitantes) taxa de internação por DM, respectivamente.

Finalmente, o Índice de Moran Local resultou no valor de $0,336(p=0,01)$, indicando, portanto, a existência de correlação espacial significativa entre as internações hospitalares por DM. Também foram identificados padrões espaciais de distribuição locais por meio do cálculo do LISA (Local Spatial Analysis) e, portanto, os valores menores que $5 \%$ foram identificados por apresentarem dependência em relação às taxas observadas nas áreas vizinhas. Diante disso, verificou-se que os municípios de Linhares, João Neiva, Fundão, Serra, Cariacica, Domingos Martins, Irupi, Ibitirama, São José do Calçado e Apiacá expressaram níveis de significância menor que $1 \%$. Ademais, outros 17 municípios espalhados entre as regiões do Estado apresentaram níveis de significância menores que $5 \%$ (Figura 2). 
Figura 2 - Distribuição espacial das taxas de internações hospitalares por diabetes mellitus em regiões com significativa dependência espacial. Espirito Santo, 2011-2015

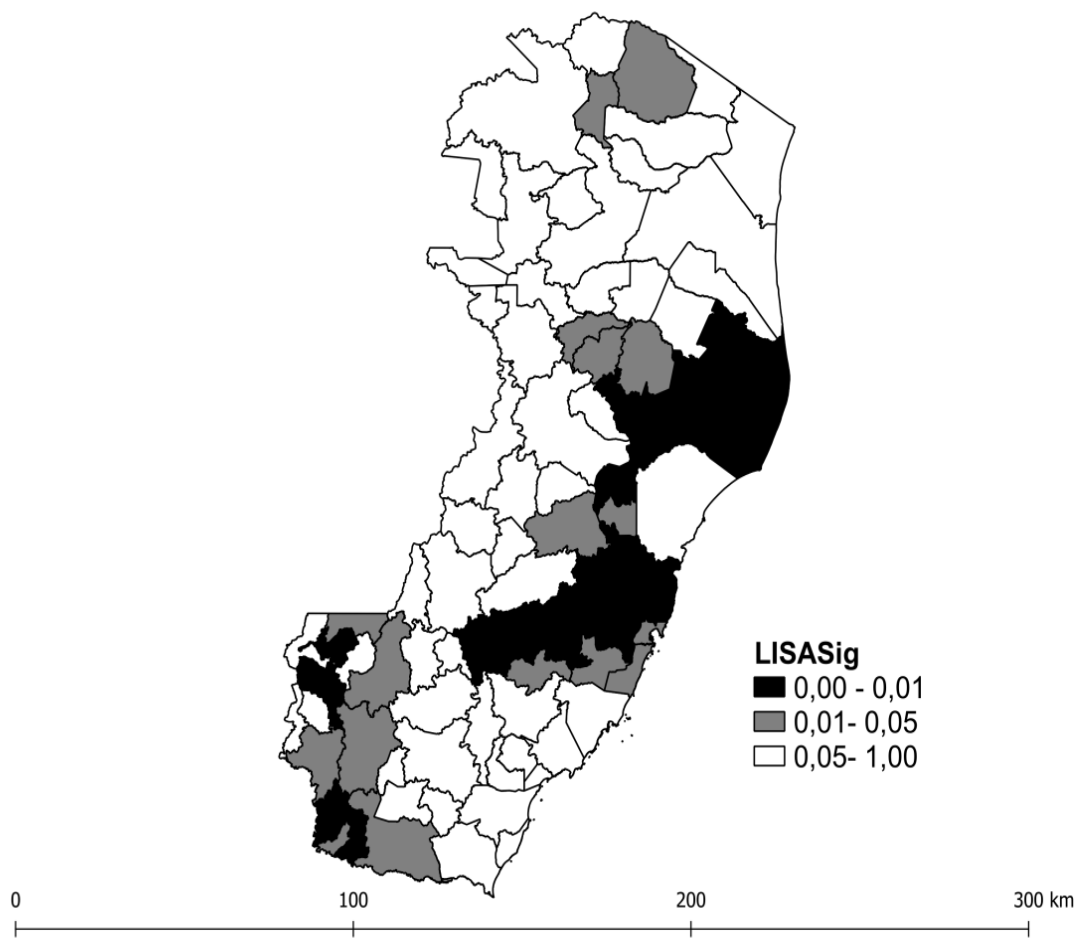

\section{DISCUSSÃO|}

A distribuição espacial das internações hospitalares por DM mostrou que os municípios de Irupi e Vitória apresentaram, respectivamente, a maior e a menor taxa de internação do ES no período de 2011 a 2015.

Ao caracterizar as regiões de saúde, observou-se que as regiões de saúde Metropolitana e Sul obtiveram a maior porcentagem de internações do Estado devido à maior concentração de serviços de alta complexidade, o que, possivelmente, gera a migração de pessoas dos municípios de menor aporte assistencial em busca de atendimento hospitalar. Além disso, possuem a maior população residente do Estado. Estudo realizado no Paraná (SC) mostrou que a maior proporção de internações por DM pode ser explicada pela maior população residente na região metropolitana de saúde onde se localizam os municípios mais populosos ${ }^{14}$.

Os resultados deste estudo ainda mostraram que no decorrer dos cinco anos estudados houve uma maior proporção de internação de mulheres diabéticas, e isso sugere maior prevalência da doença no sexo feminino ${ }^{15}$, assim como a maior procura por serviços de saúde ${ }^{16,17}$. Em consonância, no estado do Ceará, a maioria das hospitalizações por diabetes ocorreram no sexo feminino $(58,4 \%)$, entretanto, quando estratificadas por sexo, os homens apresentaram tendência crescente, e mulheres tendência decrescente ${ }^{18}$.

Em relação à raça/cor, os pardos representaram a maioria no estudo. Segundo a Sociedade Brasileira do Diabetes, as consolidações científicas indicam que a relação dessa variável ainda é discutível, não se pode confirmar uma distinção de risco para o desenvolvimento do DM entre brancos, pardos ou pretos ${ }^{19}$. No entanto, esse resultado pode ser reflexo das expressivas desigualdades sociais da maioria da população brasileira como aponta estudo no estado da Bahia e em Feira de Santana $(B A)^{20}$. Ainda, de acordo com Berlon et al. ${ }^{21}$, a maior prevalência de doenças em indivíduos não brancos sugere que um conjunto de fatores socioeconômicos, comportamentais, estruturais, acesso e uso de serviços de saúde podem produzir diferenças nas condições de saúde entre as etnias. Ressalta-se como limitação o fato de a variável raça/cor ser autodeclarada, e quase um quarto dos dados estarem sem informação. 
Quanto à faixa etária, a pesquisa apontou maior frequência de internação em indivíduos maiores de 60 anos, o que corresponde à literatura indicando que o aumento da expectativa de vida influencia na incidência da doença, igualmente crescente, de doenças crônicas degenerativas na população ${ }^{22,23,24 .}$

Além disso, pode-se observar que a prevalência de DM apresenta relação direta com o aumento da faixa etária. Essa relação também pode ser observada na Pesquisa Nacional de Saúde ${ }^{25}$ e no estudo realizado em São Paulo ${ }^{26}$ os quais mostraram que, além de alterações do metabolismo e hormonais consequentes do envelhecimento, o avanço da idade pode estar associado à redução da atividade física e aumento de hábitos alimentares pouco saudáveis.

O presente estudo também apontou para uma correlação espacial significativa entre as taxas de internações hospitalares por DM, porém uma correlação fraca em contexto estadual. Desta forma, a taxa de internação por DM de um município tende a ser mais semelhante à do município vizinho do que à de município mais distante, uma vez que as regiões vizinhas geralmente compartilham de características demográficas e socioeconômicas semelhantes ${ }^{27}$.

O aumento da incidência e prevalência do DM está diretamente relacionado à obesidade, sedentarismo, envelhecimento populacional e urbanização como também à maior sobrevida de pacientes portadores de $\mathrm{DM}^{19}$. Diante desse contexto, a frequência das complicações associadas a essa doença somadas aos desafios da Atenção Primária à Saúde, enquanto coordenadora do cuidado, resultam no aumento do número de internações hospitalares.

Dentre as limitações deste estudo, cabe destacar que a fonte dos dados (SIH-SUS) registra somente as hospitalizações financiadas pelo Sistema Único de Saúde e não permite identificar variações na oferta de leitos durante o período de análise. Por outro lado, os achados do presente estudo são relevantes para auxiliar gestores e diferentes profissionais de saúde no planejamento e implementação de ações que visem à redução das internações por DM. Ademais, a aplicação dos instrumentos de análise espacial vem aumentando no campo da saúde ${ }^{28,29,30}$, uma vez que essas técnicas permitem um diagnóstico situacional, evidenciando as regiões que necessitam de ampliação, estruturação e/ou aperfeiçoamento dos serviços de saúde.

\section{CONCLUSÃO |}

A partir da análise espacial, foi possível identificar os municípios com maiores taxas de internação por DM no Espírito Santo, ressaltando a sua importância no planejamento das ações nos serviços de atenção primária direcionadas aos grupos populacionais de risco.

As internações hospitalares por DM são causadas, em sua maioria, por complicações da patologia. Dessa forma, a análise aplicada pode ser utilizada como instrumento pelos gestores de saúde para ampliação, estruturação e aperfeiçoamento dos serviços de atenção primária à saúde a fim de prover o tratamento adequado para os pacientes diagnosticados com DM.

\section{REFERÊNCIAS |}

1. International Diabetes Federation. IDF diabetes atlas. 8. ed. Bruxelas: IDF; 2017.

2. Brasil. Ministério da Saúde. Secretaria de Atenção à Saúde. Diabetes Mellitus. Brasília: Ministério da Saúde; 2006.

3. Brasil. Ministério da Saúde. Secretaria de Vigilância em Saúde. VIGITEL Brasil 2016: estimativas sobre frequência e distribuição sociodemográfica de fatores de risco e proteção para doenças crônicas nas capitais dos 26 estados brasileiros e no Distrito Federal em 2016. Brasília: Ministério da Saúde; 2017

4. Costa AF, Flor LS, Campos MR, Oliveira AF, Costa MFS, Silva RS, et al. Carga do diabetes mellitus tipo 2 no Brasil. Cad Saúde Pública. 2017; 33(2):e00197915.

5. NCD Risk Factor Collaboration (NCD-RisC). Worldwide trends in diabetes since 1980: a pooled analysis of 751 population-based studies with 4,4 million participants. Lancet. 2016; 387(10027):1513-30.

6. Gamba A, Gotlieb SLD, Bergamaschi DP, Vianna LAC. Lower extremity amputations in diabetic patients: a casecontrol study. Rev Saúde Pública. 2004; 38(3):399-404.

7. Nyenwe EA, Jerkins TW, Umpierrez GE, Kitabchi AE. Management of type 2 diabetes: evolving strategies for the 
treatment of patients with type 2 diabetes. Metabolism. 2011; 60(1):1-23.

8. Berardis G, D’Ettorre A, Graziano G, Lucisano G, Pellegrini F, Cammarota S, et al. The burden of hospitalization related to diabetes mellitus: a populationbased study. Nutr Metab Cardiovasc Dis. 2012; 22(7):60512.

9. Pazó RG, Frauches DO, Galvêas DP, Stefenoni AV, Cavalcante ELB, Pereira-Silva FH. Internações por condições sensíveis à atenção primária no Espírito Santo: estudo ecológico descritivo no período 2005-2009. Epidemiol Serv Saúde. 2012; 21(2):275-82.

10. Alfradique ME, Bonolo PF, Dourado I, Lima-Costa MF, Macinko J, Mendonça CS, et al. Internações por condições sensíveis à atenção primária: a construção da lista brasileira como ferramenta para medir o desempenho do sistema de saúde (Projeto ICSAP - Brasil). Cad Saúde Pública. 2009; 25(6):1337-49.

11. DATASUS [Internet]. População residente: estudo de estimativas populacionais por município, idade e sexo 2000-2015 (Brasil) [acesso em 14 dez 2017]. Disponível em: URL: http://tabnet.datasus.gov.br/cgi/deftohtm. exe?novapop/cnv/popbr.def.

12. Bailey TC, Gatrell AC. Interactive spatial data analysis. Essex: Longman; 1995.

13. Anselin L. Local indicators of spatial association (LISA). Geogr Anal. 1995; 27(2):93-115.

14. Arruda GO, Schmidt DB, Marcon SS. Internações por diabetes mellitus e a Estratégia Saúde da Família, Paraná, Brasil, 2000 a 2012. Ciênc Saúde Coletiva. 2018; 23(2):54352.

15. Carstensen B, Kristensen JK, Ottosen P, Borch-Johnsen K; Steering Group of the National Diabetes Register. The danish national diabetes register: trends in incidence, prevalence and mortality. Diabetologia. 2008; 51(12):218796.

16. Silva ZP, Ribeiro MCSA, Barata RB, Almeida MF. Perfil sociodemográfico e padrão de utilização dos serviços de saúde do Sistema Único de Saúde (SUS), 2003-2008. Ciênc Saúde Coletiva. 2011; 16(9):3807-16.
17. Bercke BR, Selenguini GS, Lemos RA, Ferreira EB, Lima DB. Cuidado a partir da atenção primária: estado nutricional do portador de diabetes mellitus. Rev Universidade Vale Rio Verde. 2017; 15(1):229-39.

18. Santos FAL, Lima WP, Santos AL, Teston EF, Marcon SS. Hospitalizações por diabetes em adultos e idosos no Ceará, 2001-2012. Epidemiol Serv Saúde. 2014; 23(4):65563.

19. Sociedade Brasileira de Diabetes. Diretrizes da Sociedade Brasileira de Diabetes: 2015-2016. São Paulo: AC Farmacêutica; 2015.

20. Miranda SS, Carvalho S, Andrade TM, Coelho JMF, Gomes Filho IS. Atividade física e o controle glicêmico de pacientes com diabetes mellitus tipo II. Rev Bras Pesq Saúde. 2015; 17(3):33-40.

21. Belon AP, Francisco PMSB, Barros MBA, César CLG, Carandina L, Goldbaun M, et al. Diabetes em idosos: perfil sócio-demográfico e uso de serviços de saúde. In: Anais do 16. Encontro Nacional de Estudos Populacionais; 2008 set/out 29-03; Caxambu, Brasil. Belo Horizonte: ABEP; 2008.

22. Vasconcelos CR, Dutra DA, Oliveira EM, Fernandes S. Perfil socioeconômico e clínico de um grupo de diabéticos em tratamento hemodialítico em Curitiba. Rev Uniandrade. 2013; 14(2):183-200.

23. Santos AL, Cecílio HPM, Teston EF, Arruda GO, Peternella FMN, Marcon SS. Microvascular complications in type 2 diabetes and associated factors: a telephone survey of self-reported morbidity. Ciênc Saúde Coletiva. 2015; 20(3):761-70.

24. Ramos JS, Carvalho Filha FSS, Silva RNA. Avaliação da adesão ao tratamento por idosos cadastrados no programa do Hiperdia. Rev Gest em Sist Saúde. 2015; 4(1):29-39.

25. Iser BPM, Stopa SR, Chueiri PS, Szwarcwald CL, Malta DC, Monteiro HOC, et al. Prevalência de diabetes autorreferido no Brasil: resultados da Pesquisa Nacional de Saúde 2013. Epidemiol Serv Saúde. 2015; 24(2):305-14.

26. Francisco PMSB, Belon AP, Barros MBA, Carandina L, Alves MCGP, Goldbaum M, et al. Diabetes auto-referido 
em idosos: prevalência, fatores associados e práticas de controle. Cad Saúde Pública. 2010; 26(1):175-84.

27. Souza GOC, Torres HG. O estudo da metrópole e o uso de informações georreferenciadas. São Paulo Perspec. 2003; 17(3-4):35-44.

28. Dias BAS, Sales CMM, Bertolde AI, Maciel ELN. Análise espacial da tuberculose infantil no Espírito Santo no período de 2001 a 2011. Rev Bras Pesq Saúde. 2015; 16(3):92-8.

29. Oliveira GS, Lima MCBM, Lyra CO, Oliveira AGRC, Ferreira MAF. Desigualdade espacial da mortalidade neonatal no Brasil: 2006-2010. Ciênc Saúde Coletiva. 2013; 18(8):2431-41.

30. Macente LB, Zandonade E. Spatial distribution of suicide incidence rates in municipalities in the state of Espírito Santo (Brazil), 2003-2007: spatial analysis to identify risk areas. Rev Bras Psiquiatr. 2012; 34(3):261-9.

Correspondência para/Reprint request to:

\section{Barbara Almeida Soares Dias}

Rua Tamoyo, 92,

Glória, Vila Velha/ES, Brasil

E-mail: barbaraalmeidasd@gmail.com

Recebido em: 15/11/2018

Aceito em: 17/08/2020 